\title{
Initial Nutritional Status and Clinical Outcomes in Patients With Deep Neck Infection
}

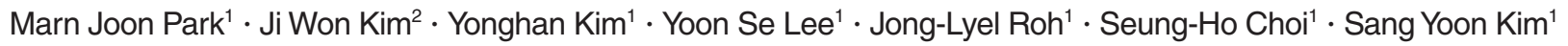 \\ Soon Yuhl Nam ${ }^{1}$
}

${ }^{l}$ Department of Otolaryngology, Asan Medical Center, University of Ulsan College of Medicine, Seoul;

${ }^{2}$ Department of Otolaryngology, Inha University College of Medicine, Incheon, Korea

Objectives. The current study aims to determine the correlation between nutritional status upon presentation and disease severity, as well as treatment and survival outcomes.

Methods. Patients who were diagnosed with deep neck infection, underwent at least one surgical drainage/debridement, and had more than 1 week of hospitalization at a tertiary medical center from 2007 to 2015 were retrospectively included.Thereafter, initial serum albumin, C-reactive protein (CRP), and body mass index (BMI) were reviewed.

Results. A total of 135 patients were included in the final analysis. Accordingly, the proportion of patients with simultaneous mediastinitis $(21.0 \%)$, necrotizing fasciitis $(12.9 \%)$, disease extent $>1$ cervical level $(72.6 \%)$, mean CRP $(22.4$ $\mathrm{mg} / \mathrm{dL}$ ), mean length of hospitalization (25.0 days), and mean 1-week follow-up CRP (7.2 $\mathrm{mg} / \mathrm{dL}$ ) was significantly higher in the hypoalbuminemia group (initial serum albumin $<3.0 \mathrm{~g} / \mathrm{dL}$ ) than in the normoalbuminemia group (all $P<0.05)$. No significant correlations had been observed according to BMI status. After adjusting for age and Charlson comorbidity index, odds ratios for the following outcomes were calculated in patients initially presenting with hypoalbuminemia: simultaneous mediastinitis (3.07), necrotizing fasciitis (7.89), disease extent $>1$ cervical level (2.12), initial serum CRP over $20 \mathrm{mg} / \mathrm{dL}$ (3.79), hospitalization of more than 14 days (4.10), 1-week follow-up CRP over 5 $\mathrm{mg} / \mathrm{dL}$ (3.78), and increased duration for an over 50\% decrease in initial CRP (2.70) (all $P<0.05)$. Although intravascular albumin replenishment decreased the proportion of patients with hypoalbuminemia after 2 weeks $(P<0.05)$, it did not significantly predict better treatment outcomes.

Conclusion. Among the markers reflecting an individual's nutritional state, an initial serum albumin of less than $3.0 \mathrm{~g} / \mathrm{dL}$ was an independent serologic marker predicting increased disease severity and complications in patients with deep neck infection.

Keywords. Retrophnarynx Abscess; Fasciitis; Necrotizing; Hypoalbuminemia; Malnutrition; Drainage

\section{INTRODUCTION}

Prompt assessment and selection of patients in need for surgical intervention should be the most crucial initial treatment strategy in the management of deep neck infections, as previously em-

- Received January 24, 2018

Revised April 22, 2018

Accepted May 28, 2018

- Corresponding author: Soon Yuhl Nam

Department of Otolaryngology, Asan Medical Center, University of Ulsan College of Medicine, 88 Olympic-ro 43-gil, Songpa-gu, Seoul 05505, Korea Tel: +82-2-3010-3710, Fax: +82-2-489-2773

E-mail: synam@amc.seoul.kr phasized by many surgeons [1,2]. Infectious and inflammatory conditions of the human neck can cause rapid airway compromise [3]. Furthermore, the spread of infection through the prevertebral fascia could result in simultaneous mediastinitis or osteomyelitis of the cervical spine, which could be catastrophic for patient survival [4]. However, many physicians have argued that performing only physical examination of the neck is insufficient for the accurate assessment of severity and prediction of prognosis in patients with deep neck infection [5].

In 2004, Wong et al. [6] proposed a scoring system for predicting prognosis in necrotizing cervical fasciitis that included white blood cells, hemoglobin A1C, serum sodium, glucose, cre-

Copyright (C) 2018 by Korean Society of Otorhinolaryngology-Head and Neck Surgery.

This is an open-access article distributed under the terms of the Creative Commons Attribution Non-Commercial License (http://creativecommons.org/licenses/by-nc/4.0)

which permits unrestricted non-commercial use, distribution, and reproduction in any medium, provided the original work is properly cited. 
atinine, and C-reactive protein (CRP). Their scoring system, termed Laboratory Risk Indicator for Necrotizing Fasciitis (LRINEC), was the first attempt to predict severe types of infections in the cervical area utilizing initial laboratory markers. However, the LRINEC scoring system showed weak predictability in cases where infections were exclusively limited to the deeper spaces of the neck $[7,8]$.

In terms of critical care, low serum albumin levels were associated with poorer clinical outcomes and lower survival $[9,10]$. According to Vincent et al. [11], serum albumin level was an independent predictor of prognosis and treatment response in patients with acute distress. Additionally, in 2015, Moon [5] proposed a modified LRINEC scoring system that added initial serum albumin levels to the conventional LRINEC scoring system. Compared to the conventional scoring system, the modified LRINEC scores were shown to have higher modality in predicting clinical outcomes.

Serum albumin and body mass index (BMI) are widely accepted, easily measured indicators reflecting the overall nutritional status of an individual [12-14]. Therefore, the current study aimed to (1) compare the initial severity of deep neck infections between well-and poorly-nourished patients, (2) determine the risk for poorer clinical presentation and clinical outcomes, and (3) determine whether albumin replenishment could improve clinical outcomes.

\section{MATERIALS AND METHODS}

\section{Study population}

Study subjects were recruited through retrospective medical chart review. Patients who primarily visited Asan Medical Center (a tertiary medical center) via the outpatient clinic or emergency room and were subsequently diagnosed with deep neck infection from January 2007 to December 2015 were reviewed. Patients with deep neck infection were defined as those who had undergone at least one surgical drainage/debridement of the infection source in addition to being hospitalized for more than 7 days. Patients under the age of 18 and those with deep cervical infection due to underlying malignancy, tuberculosis, trauma, and foreign material insertion to the head and neck area were

\section{H I G G H L I I G H T T S}

- Risk factors of complicated deep neck infections were retrospectively examined in 135 patients.

- Initial serum albumin less than $3.0 \mathrm{~g} / \mathrm{dL}$ was an independent serologic marker to predict complicated deep neck infection $(P<0.05)$.

- Intravenous albumin replenishment did not show a significance in the prediction of better treatment outcomes. excluded. Additionally, patients who had undergone contrastenhanced computed tomography (ceCT) or surgical debridement in other medical centers, as well as those referred from other medical centers, were excluded. This study was approved by the Institutional Review Board of Asan Medical Center (IRB No. 2017-0031).

\section{Definition and categorization of deep neck infection}

All patients underwent ceCT of the head and neck area, as well as the chest area in cases where infectious spread along the mediastinum was evident. The diagnosis of deep neck infection in each patient was confirmed by an official reading from a boardcertified radiologist. Furthermore, two sub-categories of deep neck infections were established. Accordingly, patients whose ceCT showed an evident abscess pocket(s) in the deep cervical space were defined as the "deep cervical abscess" group, whereas those suffering from severe necrosis with soft-tissue infection spreading along the deep cervical fascia and cervical muscles were defined as the "necrotizing fasciitis" group. Abscess pockets exclusively located at the peritonsillar space, oropharyngeal/ laryngeal mucosa, and salivary glands were not considered as true "deep neck infections" and therefore not included in the study.

\section{Management of deep neck infection}

Each patient had undergone surgical debridement followed by hospital admission. Decisions regarding patient treatment were made by a board-certified head and neck surgeon. Upon initial patient encounter, prompt resuscitation of vital signs, securing of the upper airway, and peripheral blood laboratory/microbiology tests had been performed. Intravenous (IV) antibiotics were immediately administered empirically based on established guidelines. Upon surgical drainage of deep cervical abscesses, a paired swab and tissue culture had been obtained for the identification of underlying microorganisms. Thereafter, the regimen and dosage of antibiotic agents were modified based on the results of the bacterial culture study and consultations with an infectious disease specialist. Cases where in the infection had spread to the mediastinum through the prevertebral space were consulted with a cardiothoracic surgeon, and surgical intervention and drainage of pus were performed as needed. Some patients with severe bacteremia and sepsis despite adequate surgical and medical treatment needed aggressive intervention, including critical patient care in intensive care unit admission. In cases with persisting hypoalbuminemia, single-to-multiple IV administrations of $100 \mathrm{~mL}$ of $20 \%$ albumin solution were considered. However, this could only be performed upon the informed consent from the patient or their guardians. Patients were discharged when they showed no evidence of active infection/inflammation and were tolerant to reasonable amounts of oral feeding. 


\section{Measured variables}

Demographic information (age and sex) was reviewed. The severity of the underlying comorbidity in each patient was evaluated using the Charlson comorbidity index (CCI) in addition to reviewing the patients' medical history of hypertension, diabetes, chronic liver disease, chronic pulmonary disease, end-stage/chronic renal disease, and coronary artery disease. The ceCT images were used to determine the extent of involvement (unilateral/ bilateral), number of involved cervical levels, and presence of simultaneous mediastinitis. Peripheral blood laboratory results, including a complete blood count, chemical battery, and CRP, were available for all patients. Follow-up for the complete blood count, chemical battery, and CRP was done daily or every other day for the first and second week of treatment. For the remaining hospitalization period, blood tests were performed twice a week. Initial serum albumin and BMI were used to assess the nutritional state of each patient. Moreover, 1-week follow-up CRP and the duration for an over $50 \%$ decrease in the initial CRP were used to determine responses to treatment. Clinical outcomes were evaluated using the total number of surgical drainage, total length of hospitalization, and overall survival.

\section{Statistical analysis}

Values for or proportion of patients with each clinical factor according to the initial and 2-week nutritional status were compared using Student $t$-test, chi-square test, and Fisher exact test. Odds ratios (ORs) for the development of each clinical manifestation and worse clinical outcomes with poorer nutritional status, older age, and poorer underlying conditions were calculated using univariate logistic regression analysis. Multivariate analysis of the ORs for each clinical result was performed after adjusting for age and CCI. Survival curves were plotted using the KaplanMeier method, while the log-rank test was used to compare overall survival and elapsed time for a $50 \%$ reduction in serum CRP according to initial serum albumin levels. All of the tests were two-sided, and a $P$-value $<0.05$ was considered statistically significant. Statistical analyses were performed using IBM SPSS ver. 21.0 (IBM Corp., Armonk, NY, USA).

\section{RESULTS}

\section{Patient characteristics}

A total of 135 patients satisfied the inclusion criteria for the current study (Table 1), among which 50 (31.6\%) and 85 (53.8\%) accounted for males and females, respectively, with a median age of 62 years. Accordingly, 19 patients (14.1\%) had hypertension, 46 (34.1\%) diabetes, four (3.0\%) chronic hepatitis, five (3.7\%) end-stage renal disease (ESRD), two (1.5\%) chronic pulmonary disease, and $19(14.1 \%)$ coronary artery disease, resulting in 73 patients $(54.1 \%)$ with a CCI $\geq 1$. The median serum albumin level upon initial presentation was $3.1 \mathrm{~g} / \mathrm{dL}$ with $62 \mathrm{pa}-$
Table 1. Patient characteristics

\begin{tabular}{lc}
\hline Characteristics & Value $(\mathrm{n}=135)$ \\
\hline Sex & $50(31.6): 85(53.8)$ \\
Male:female & $62(51-74)$ \\
Age $(\mathrm{yr})$ & \\
Charlson comorbidity index & $62: 40: 25: 8: 0$ \\
0:1:2:3:6 & $(45.9: 29.6: 18.5: 5.9: 0)$ \\
& \\
Nutritional markers on initial presentation & $6.5(5.6-7.2)$ \\
Serum total protein (g/dL) & $3.1(2.5-3.6)$ \\
Serum albumin (g/dL) & $23.0(20.8-25.8)$ \\
Body mass index (kg/m²) & $105(66.5): 30(19.0)$ \\
Extent of deep neck infection & $85(63.0)$ \\
Unilateral:bilateral & $126(93.3): 9(6.7)$ \\
Involvement in more than 1 cervical level & $19(14.1)$ \\
Deep cervical abscess:necrotizing fasciitis & \\
Presence of simultaneous mediastinitis & \\
Clinical outcome & \\
No. of surgical drainage & $76: 38: 14: 3: 2: 0: 1$ \\
1:2:3:4:5:6:7 & $14(10-23)$ \\
Length of hospitalization (day) & $11(8.1)$ \\
Death during treatment & $(56.3: 28.1: 10.4: 3.0: 1.5: 0: 0.7)$ \\
\hline
\end{tabular}

Values are presented as number (\%) or median (range).

tients $(45.9 \%)$ having initial hypoalbuminemia (serum albumin $<3.0 \mathrm{~g} / \mathrm{dL}$ ). Moreover, the median BMI value was $23.0 \mathrm{~kg} / \mathrm{m}^{2}$ with 17 patients $(12.6 \%)$ being underweight (BMI $<18.5 \mathrm{~kg} /$ $\left.\mathrm{m}^{2}\right)$. A total of 30 patients $(19.0 \%)$ showed bilateral disease involvement, while $85(63.0 \%)$ had an infection that extended to more than one cervical level. The ceCT revealed a definite abscess pocket in 126 patients $(93.3 \%)$ and necrotizing fasciitis in nine $(6.7 \%)$. Simultaneous mediastinitis was observed in 19 patients $(14.1 \%)$. The mean number of surgical drainage was 1.7 (standard deviation, 1.0) with a minimum of 1 and a maximum of 7 , while the median duration of hospitalization was 14 days. Despite aggressive resuscitation and adequate control of the infection source, 11 patients $(8.1 \%)$ died from sepsis followed by multiple organ failure.

\section{Correlation between initial nutritional status and clinical manifestations}

Values for and proportion of patients with each clinical finding were compared according to initial serum albumin levels and BMI (Table 2). The proportion of patients with older age, simultaneous mediastinitis, necrotizing fasciitis, disease extent of more than one cervical level, and higher initial CRP was significantly greater in the hypoalbuminemia group than in the normoalbuminemia group (all $P<0.05$ ). The hypoalbuminemia group also showed poorer treatment responses and clinical outcomes, which resulted in longer duration of hospitalization, higher 1-week follow-up CRP, and longer time interval for a $>50 \%$ decrease in the initial CRP level (all $P<0.05$ ). However, the 
number of surgical drainage and overall mortality rate did not differ between the two groups. On the other hand, no significant differences were found between the underweight and normalto-overweight BMI groups. ORs showed that individuals with initial serum albumin levels below $3.0 \mathrm{~g} / \mathrm{dL}$ had a significant increase in the risk of developing simultaneous mediastinitis (2.96), necrotizing fasciitis (10.67), disease extent of more than one cervical level (2.18), and initial serum CRP over $20 \mathrm{mg} / \mathrm{dL}$ (4.20) (all $P<0.05)$ (Table 3). In terms of clinical outcomes, the hypoalbuminemia group showed a significant increase in the risk for hospitalization of more than 14 days (4.90), 1-week followup CRP over $5 \mathrm{mg} / \mathrm{dL}$ (3.43), and longer time interval for an over $50 \%$ decrease in the initial CRP (3.10) (all $P<0.05)$. Multivariate analysis showed significantly increased OR for more severe initial presentation and worse clinical outcomes as well (Table 4). To determine possible confounding clinical variables, univariate analysis was conducted for patients over 70 and those having a CCI $>1$ (Supplementary Table 1 ). In the elderly group (age $>70$

Table 2. Differences in clinical manifestations according to nutritional status

\begin{tabular}{|c|c|c|c|c|c|c|}
\hline Patient demographics & $\begin{array}{l}\text { Hypoalbuminemia } \\
\text { (serum albumin } \\
<3.0 \mathrm{~g} / \mathrm{dL}, \mathrm{n}=62 \text { ) }\end{array}$ & $\begin{array}{c}\text { Normoalbuminemia } \\
\text { (serum albumin } \\
\geq 3.0 \mathrm{~g} / \mathrm{dL}, \mathrm{n}=73 \text { ) }\end{array}$ & $P$-value ${ }^{a)}$ & $\begin{array}{c}\text { Underweight } \\
\left(\mathrm{BMI}<18.5 \mathrm{~kg} / \mathrm{m}^{2},\right. \\
\mathrm{n}=17)\end{array}$ & $\begin{array}{c}\text { Normal-to-overweight } \\
\left(\mathrm{BMI} \geq 18.5 \mathrm{~kg} / \mathrm{m}^{2}\right. \\
\mathrm{n}=118)\end{array}$ & $P$-value ${ }^{a)}$ \\
\hline Age (yr) & $67.8 \pm 13.3$ & $56.1 \pm 15.8$ & $<0.001^{\text {b) }}$ & $59.6 \pm 20.2$ & $61.7 \pm 15.1$ & 0.612 \\
\hline $\mathrm{CCl}>1$ & $37(59.7)$ & $36(49.3)$ & 0.229 & $8(47.1)$ & $65(55.1)$ & 0.535 \\
\hline \multicolumn{7}{|l|}{$\begin{array}{l}\text { Clinical \& laboratory findings on initial } \\
\text { presentation }\end{array}$} \\
\hline Simultaneous mediastinitis & $13(21.0)$ & $6(8.2)$ & $0.034^{\mathrm{b})}$ & $4(23.5)$ & $15(12.7)$ & 0.261 \\
\hline Necrotizing fasciitis & $8(12.9)$ & $1(1.4)$ & $0.012^{\mathrm{b})}$ & 0 & $9(7.6)$ & 0.602 \\
\hline Bilateral involvement & $17(27.4)$ & $13(17.8)$ & 0.181 & $4(23.5)$ & $26(22.0)$ & 1.000 \\
\hline Extended to $>1$ cervical level & $45(72.6)$ & $40(54.8)$ & $0.033^{\mathrm{b})}$ & $10(58.8)$ & $75(63.6)$ & 0.705 \\
\hline WBC $\left(\times 10^{6} / L\right)$ & $10,600.3 \pm 7,550.6$ & $9,164.5 \pm 6,872.5$ & 0.445 & $12,727.2 \pm 4,784.0$ & $11,943.8 \pm 4,402.0$ & 0.499 \\
\hline $\mathrm{CRP}(\mathrm{mg} / \mathrm{dL})$ & $22.4 \pm 9.8$ & $17.4 \pm 8.8$ & $0.002^{\mathrm{b})}$ & $18.5 \pm 11.4$ & $19.9 \pm 9.3$ & 0.578 \\
\hline \multicolumn{7}{|l|}{$\begin{array}{l}\text { Clinical outcome and response to the } \\
\text { treatment }\end{array}$} \\
\hline Length of hospitalization (day) & $25.0 \pm 14.6$ & $14.6 \pm 10.9$ & $<0.001^{\mathrm{b})}$ & $21.1 \pm 18.7$ & $19.1 \pm 12.1$ & 0.584 \\
\hline No. of surgical drainage & $1.8 \pm 1.2$ & $1.6 \pm 0.8$ & 0.248 & $1.8 \pm 0.9$ & $1.7 \pm 1.0$ & 0.716 \\
\hline No. of dead patients & $4(6.5)$ & $7(9.6)$ & 0.548 & $1(5.9)$ & $10(8.5)$ & 1.000 \\
\hline 1-Week follow-up CRP (mg/dL) & $7.2 \pm 5.2$ & $4.4 \pm 4.6$ & $0.001^{\mathrm{b})}$ & $6.3 \pm 6.2$ & $5.6 \pm 4.9$ & 0.547 \\
\hline $\begin{array}{l}\text { Time interval for } 50 \% \text { decrease of initial } \\
\text { CRP (day) }\end{array}$ & $6.4 \pm 4.2$ & $5.0 \pm 3.0$ & $0.029^{b)}$ & $6.1 \pm 3.9$ & $5.5 \pm 3.6$ & $0.669^{b)}$ \\
\hline
\end{tabular}

Values are presented as mean \pm standard deviation or number (\%).

$\mathrm{BMI}$, body mass index; CCI, Charlson comorbidity index; WBC, white blood cell; CRP, C-reactive protein.

a) Values for or proportion of patients in each clinical factor between the two groups (hypoalbuminemia vs. normoalbuminemia group and underweight vs. normal-to-overweight group) upon initial presentation were compared using Student $t$-test, chi-squared test, and Fisher exact test. ${ }^{\text {b) }} P$-value $<0.05$.

Table 3. Univariate analyses of nutritional markers according to initial clinical presentation, treatment response, and clinical outcomes

\begin{tabular}{|c|c|c|c|c|}
\hline \multirow{2}{*}{ Variable } & \multicolumn{2}{|c|}{ Hypoalbuminemia (serum albumin $<3.0 \mathrm{~g} / \mathrm{dL}$ ) } & \multicolumn{2}{|c|}{ Underweight $\left(\mathrm{BMl}<18.5 \mathrm{~kg} / \mathrm{m}^{2}\right)$} \\
\hline & OR $(95 \% \mathrm{CI})^{a)}$ & $P$-value & OR $\left.(95 \% \mathrm{Cl})^{2}\right)$ & $P$-value \\
\hline \multicolumn{5}{|l|}{ Initial presentation } \\
\hline Simultaneous mediastinitis & $2.96(1.05-8.34)$ & $0.040^{b)}$ & $2.11(0.61-7.34)$ & 0.239 \\
\hline Necrotizing fasciitis & $10.67(1.30-87.86)$ & $0.028^{b)}$ & $1.29(0.10-1.34)$ & 0.989 \\
\hline Bilateral involvement & $1.74(0.77-3.96)$ & 0.183 & $1.09(0.33-3.62)$ & 0.890 \\
\hline Extended to $>1$ cervical level & $2.18(1.06-4.50)$ & $0.034^{\text {b) }}$ & $0.82(0.29-2.31)$ & 0.706 \\
\hline Serum CRP > 20 mg/dL & $4.20(2.04-8.65)$ & $<0.001^{\mathrm{b})}$ & $1.13(0.41-3.13)$ & 0.817 \\
\hline \multicolumn{5}{|l|}{ Clinical outcomes and treatment response } \\
\hline Length of hospitalization $>14$ days & $4.90(2.33-10.28)$ & $<0.001^{b)}$ & $0.95(0.34-2.63)$ & 0.920 \\
\hline No. of surgical drainage $>1$ & $1.26(0.64-2.49)$ & 0.508 & $1.53(0.55-4.24)$ & 0.414 \\
\hline Death & $1.65(0.18-4.33)$ & 0.509 & $0.68(0.08-5.63)$ & 0.717 \\
\hline 1-Week follow-up CRP > 5 mg/dL & $3.43(1.68-7.01)$ & $0.001^{b)}$ & $1.25(0.45-3.47)$ & 0.666 \\
\hline Time interval for $50 \%$ decrease of initial CRP $>7$ days & $3.10(1.36-7.08)$ & $0.007^{\mathrm{b})}$ & $1.84(0.62-5.43)$ & 0.271 \\
\hline
\end{tabular}

$\mathrm{OR}$, odds ratio; $\mathrm{Cl}$, confidence interval; $\mathrm{BMI}$, body mass index; $\mathrm{CRP}, \mathrm{C}$-reactive protein.

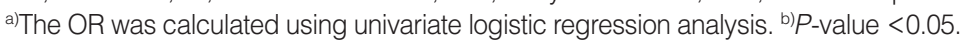


years), ORs suggested a significant increase in risk for necrotizing fasciitis (4.30), initial CRP > $20 \mathrm{mg} / \mathrm{dL}(2.10),>14$ days of hospitalization (3.11), and $>1$ week for a $>50 \%$ decrease in the initial CRP (2.26). On the contrary, no significant increase/decrease in risk was observed for patients with $\mathrm{CCI}>1$. Patients diagnosed with hepatic or renal failure showed a significant increase in the risk for necrotizing fasciitis (2.13), mediastinitis (2.16), and CRP > $20 \mathrm{mg} / \mathrm{dL}$ (4.06) but not for clinical outcomes. Subsequently, a log-rank test was conducted for overall survival and time interval for a $>50 \%$ decrease in initial CRP according to initial serum albumin levels, both of which showed worse outcomes and slower responses to treatment in the hypoalbuminemia group (both $P<0.05$ ) (Fig. 1).

Table 4. Multivariate analyses ${ }^{\text {a) }}$ of hypoalbuminemia according to initial clinical presentation, treatment response, and clinical outcome

\begin{tabular}{|c|c|c|}
\hline \multirow[t]{2}{*}{ Variable } & \multicolumn{2}{|c|}{$\begin{array}{l}\text { Hypoalbuminemia } \\
\text { (serum albumin }<3.0 \mathrm{~g} / \mathrm{dL} \text { ) }\end{array}$} \\
\hline & $\mathrm{OR}(95 \% \mathrm{Cl})^{\mathrm{b})}$ & $P$-value \\
\hline \multicolumn{3}{|l|}{ Initial presentation } \\
\hline Simultaneous mediastinitis & $3.07(1.04-9.08)$ & $0.043^{c)}$ \\
\hline Necrotizing fasciitis & $7.89(0.91-68.05)$ & $0.060^{c)}$ \\
\hline Bilateral involvement & $1.65(0.71-3.88)$ & 0.248 \\
\hline Extended to $>1$ cervical level & $2.12(1.08-4.50)$ & $0.049^{c)}$ \\
\hline Serum CRP > 20 mg/dL & $3.79(1.80-7.98)$ & $<0.001^{c)}$ \\
\hline \multicolumn{3}{|l|}{ Clinical outcomes and treatment response } \\
\hline Length of hospitalization $>14$ days & $4.10(1.91-8.83)$ & $<0.001^{c)}$ \\
\hline No. of surgical drainage $>1$ & $1.22(0.60-2.59)$ & 0.579 \\
\hline Death & $1.52(0.13-5.99)$ & 0.833 \\
\hline 1-Week follow-up CRP > 5 mg/dL & $3.78(1.76-8.10)$ & $0.001^{c)}$ \\
\hline $\begin{array}{l}\text { Time interval for } 50 \% \text { decrease of initial } \\
\text { CRP }>5 \text { days }\end{array}$ & $2.70(1.15-6.34)$ & $0.023^{c)}$ \\
\hline
\end{tabular}

$\mathrm{OR}$, odds ratio; $\mathrm{Cl}$, confidence interval; CRP, C-reactive protein.

a)Patients' age and Charlson comorbidity index were adjusted during multivariate analysis. ${ }^{\mathrm{b}}$ The $\mathrm{OR}$ was calculated using multivariate logistic regression analysis. ${ }^{c} P$-value $<0.05$.

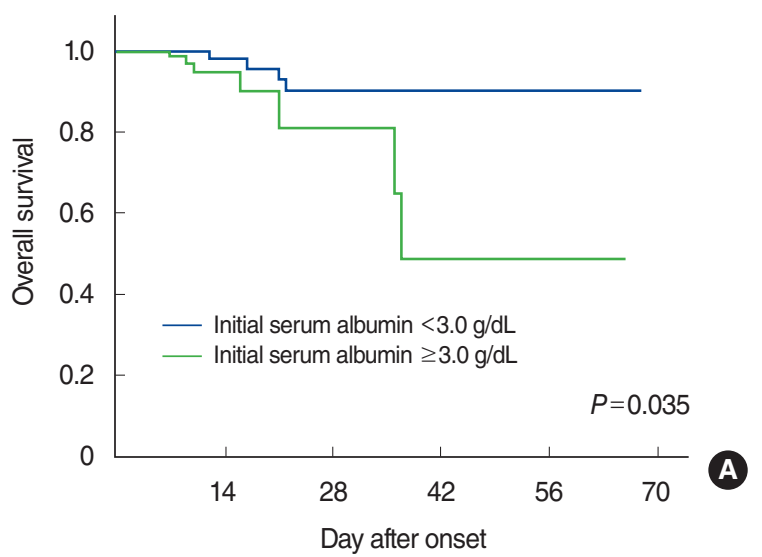

\section{Correlation between nutritional replenishment and clinical outcomes}

A total of 73 patients hospitalized for longer than 14 days were assessed for their 2-week follow-up serum albumin levels and were subsequently categorized based on such findings. Hypoalbuminemia was observed in 41 patients $(56.2 \%)$, whereas 32 $(43.8 \%)$ had serum albumin levels greater than or equal to $3 \mathrm{~g} / \mathrm{dL}$ (Supplementary Table 2). Despite the significantly higher proportion of patients receiving IV albumin replenishment in the normoalbuminemia group than in the hypoalbuminemia group $(P<$ 0.05), no significant differences in the demographic or clinical outcome parameters had been observed (Supplementary Table 2). In addition, IV albumin replenishment resulted in a 3.44-fold increase in the OR for 2-week normoalbuminemia and a 2.44fold increase in the OR for less than 30 days of hospitalization (both $P<0.05)$. However, ORs for overall survival and number of surgical drainage were not significant (Supplementary Table 3).

\section{DISCUSSION}

Based on results from 135 patients with deep neck infection, the present study showed that patients with initial hypoalbuminemia were likely to present with more severe clinical features and worse treatment outcomes. Although age over 70 years was another factor related to worse clinical manifestations, BMI and CCI were not. Patients who presented with serum albumin levels less than $3.0 \mathrm{~g} / \mathrm{dL}$ were likely to have simultaneous mediastinitis, necrotizing fasciitis, greater extent of infection, higher initial CRP, delayed treatment response, and longer duration of hospitalization. Moreover, although IV albumin replenishment was able to decrease the proportion of patients with hypoalbuminemia after 2 weeks, it was not significant in the prediction of better treatment outcomes.

The anatomy of the deep cervical space is quite complicated

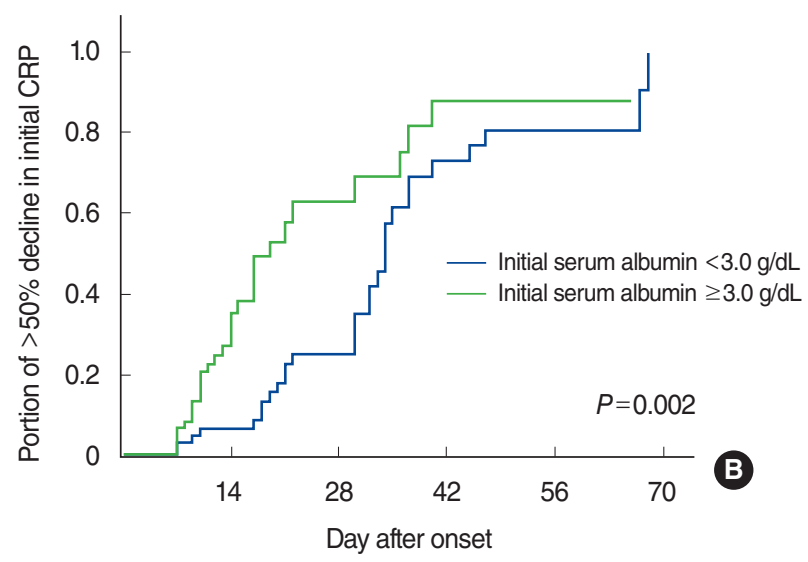

Fig. 1. Overall survival and response to treatment according to the initial serum albumin level. Kaplan-Meier curves estimating the overall survival (A) and more than 50\% reduction in initial serum C-reactive protein (CRP) (B), suggesting lower treatment responses and worse clinical outcomes in patients with initial hypoalbuminemia $(P<0.05$, both). Log-rank test, $P<0.05$. 
given that it is surrounded by multiple layers of cervical fasciae [15]. Therefore, identifying infections in the deep cervical space remains challenging, especially during the early localized stage [16]. In addition, it is even harder to accurately assess the extent and severity of infections, which is essential in making prompt decisions regarding surgical intervention versus observation with IV antibiotics [17]. Given the risk for the spread of infection to the mediastinal cavity or vertebral space, many surgeons perform surgical drainage in most of the severe cases $[18,19]$. Boscolo-Rizzo et al. [20] had reported that among 365 patients with deep neck infections, $38.1 \%$ had to undergo surgical intervention, while $18.4 \%$ developed complications despite adequate therapy.

Therefore, many previous studies have focused on assessing the severity of deep neck infections through initial laboratory findings and radiologic results to determine the validity of surgical intervention [21-28]. In 2000, Wall et al. [17] were the first to report on the validity of serologic markers in diagnosing necrotizing fasciitis. Thereafter, Wong et al. [6] established a systemized scoring scale based on the results of the initial serologic study. However, the patients included in the aforementioned studies comprised a mixture of those diagnosed with deep neck infection, cellulitis, or relatively simple abscesses, e.g., peritonsillar abscesses, thereby limiting its application for patients with deep neck infections. Thomas and Meyer [29] had utilized multiple serum laboratory markers to determine the presence of deep cervical abscesses and necrotizing fasciitis in deep neck infection cases that had no potential for prediction of necrosis due to the small portion of necrotizing fasciitis among patients infected.

In 2012, Tsai et al. [30] had published an article showing that serum albumin levels were able to predict the prognosis of necrotizing fasciitis following surgical debridement, very similar to the results presented herein. Moon's study [5], which included serum albumin in the LRINEC scoring system, showed increased scores for patients with necrotizing fasciitis which was significant. The present study showed a significantly high proportion of patients with necrotizing fasciitis in the low albumin group and that an initial serum albumin of $<3.0 \mathrm{~g} / \mathrm{dL}$ was an independent risk factor for an 8 -fold increase in necrotizing fasciitis $(P<0.05)$. These results are consistent with those from Moon's study, thereby confirming that baseline nutritional state is somewhat related to the pathophysiology of disease. BMI, on the other hand, was not correlated with severity of disease or worse clinical outcomes.

In patients with chronic hepatitis or ESRD, where in protein loss or synthesis is taking place, we observed a 2- to 4-fold increase in the OR for necrotizing fasciitis, mediastinitis, and CRP above $20 \mathrm{mg} / \mathrm{dL}$ (all $P<0.05$ ) but not for clinical outcomes. Although the percentage of patients with decreased hepatic/renal function were considerably low (8\%), the important role of albumin itself in potentially regulating the immunologic and in- flammatory response becomes clearer, resulting in altered clinical outcomes when insufficient. Interestingly, 2-week serum albumin and IV albumin replenishment were not related to clinical outcomes. In similar fashion, administration of IV albumin had no benefit on survival and outcome in patients with septic shock. Together, these findings suggest that decreased serum albumin could be responsible for weakening the host's immune system, thereby allowing rapid initial disease progression leading to a more severe and extensive state.

As proven in many previous studies, albumin plays a critical role in mediating inflammatory response, especially during the acute phase $[9,11]$. In response to systemic inflammation, the extravasation of albumin due to the increased vascular permeability leads to hypoalbuminemia in addition to the rapid breakdown of amino acids, further contributing to a decline in serum albumin levels [31]. When administered intravascularly, albumin has its own function in terms of regulating enzymatic activity, acidity, and electrolyte balance, thereby maintaining homeostasis in our body [10]. Hypoalbuminemia is thought to be both a cause and result of systemic inflammation, resulting in worsening and progression of the inflammatory reaction [32]. On the other hand, albumin is an important molecule in maintaining the osmotic pressure in the intravascular space [11]. A decline in the intravascular albumin can lead to edematous conditions in many organs, resulting in severe complications other than the infection itself, such as pneumonia or pleural effusion [33]. Together, these findings support the ability of initial serum albumin to predict prognosis and initial disease extent. Although a randomized control trial had been conducted on aggressive parenteral nutritional support during the acute phase of inflammation, its results did not show improvement in outcomes [34]. Therefore, we believe that initial serum albumin is a valid tool for assessing disease severity and predicting clinical outcomes; however, manipulation of serum albumin levels does not bring promising treatment results.

Although our study confirmed the clinical validity of initial serum albumin levels in predicting more severe disease and poorer clinical outcomes, we do acknowledge the following study limitations: (1) due to the retrospective study design, we were not able to obtain other laboratory and physical markers that represent an individual's nutritional status; (2) IV albumin replenishment had not been uniformly administered in terms of dosage, duration, and formula; (3) the lack of a previously established nutritional parameter (e.g., Malnutrition Universal Screening Tool or Nutritional Risk Screening 2002 scoring system), which could have allowed for a more comprehensive evaluation of nutritional status compared to the use of only serum albumin levels and BMI [35-37]. Therefore, we suggest the need for future prospective studies that accurately assess initial nutritional status, including a full-nutrition battery (e.g., folate, selenium, cobalamin, and arm circumference measurement), and utilize established clinical protocols for IV albumin replenishment. 
Initial serum albumin levels in patients presenting with deep neck infection was an independent serologic marker predicting more severe and complicated disease states. Additionally, initial serum albumin less than $3.0 \mathrm{~g} / \mathrm{dL}$ was an independent factor predicting worse clinical outcomes, such as longer hospitalizations and slower responses to treatment. Although IV albumin replenishment was able to correct hypoalbuminemia, it did not lead to better clinical outcomes. Our results indicate that patients with deep neck infections who present with hypoalbuminemia should be evaluated and managed more cautiously.

\section{CONFLICT OF INTEREST}

No potential conflict of interest relevant to this article was reported.

\section{ACKNOWLEDGMENTS}

The authors would like to show a gratitude to all medical staffs who participated in the treatment of the patients included for the study by offering their best professional care.

\section{SUPPLEMENTARY MATERIALS}

Supplementary materials can be found via https://doi.org/10. 21053/ceo.2018.00108.

\section{REFERENCES}

1. Brito TP, Hazboun IM, Fernandes FL, Bento LR, Zappelini CE, Chone CT, et al. Deep neck abscesses: study of 101 cases. Braz J Otorhinolaryngol. 2017 May-Jun;83(3):341-8.

2. Vieira F, Allen SM, Stocks RM, Thompson JW. Deep neck infection. Otolaryngol Clin North Am. 2008 Jun;41(3):459-83.

3. Kataria G, Saxena A, Bhagat S, Singh B, Kaur M, Kaur G. Deep neck space infections: a study of 76 cases. Iran J Otorhinolaryngol. 2015 Jul;27(81):293-9.

4. Mazzella A, Santagata M, Cecere A, La Mart E, Fiorelli A, Tartaro G, et al. Descending necrotizing mediastinitis in the elderly patients. Open Med (Wars). 2016 Nov;11(1):449-60.

5. Moon JH. Usefulness of the modified LRINEC score in the treatment of patient with deep neck infection. Korean J Otorhinolaryngol Head Neck Surg. 2015 Feb;58(2):115-9.

6. Wong CH, Khin LW, Heng KS, Tan KC, Low CO. The LRINEC (Laboratory Risk Indicator for Necrotizing Fasciitis) score: a tool for distinguishing necrotizing fasciitis from other soft tissue infections. Crit Care Med. 2004 Jul;32(7):1535-41.

7. Wong $\mathrm{CH}$, Khin LW. Clinical relevance of the LRINEC (Laboratory Risk Indicator for Necrotizing Fasciitis) score for assessment of early necrotizing fasciitis. Crit Care Med. 2005 Jul;33(7):1677.

8. Wong $\mathrm{CH}$, Wang YS. The diagnosis of necrotizing fasciitis. Curr Opin Infect Dis. 2005 Apr;18(2):101-6.
9. Casaer MP,Van den Berghe G. Nutrition in the acute phase of critical illness. N Engl J Med. 2014 Mar;370(13):1227-36.

10. Shaw JH, Koea JB. Metabolic basis for management of the septic surgical patient. World J Surg. 1993 Mar-Apr;17(2):154-64.

11. Vincent JL, Dubois MJ, Navickis RJ, Wilkes MM. Hypoalbuminemia in acute illness: is there a rationale for intervention? A meta-analysis of cohort studies and controlled trials. Ann Surg. 2003 Mar;237(3): 319-34.

12. Blackburn GL, Bistrian BR, Maini BS, Schlamm HT, Smith MF. Nutritional and metabolic assessment of the hospitalized patient. JPEN J Parenter Enteral Nutr. 1977;1(1):11-22.

13. Keys A, Fidanza F, Karvonen MJ, Kimura N, Taylor HL. Indices of relative weight and obesity. Int J Epidemiol. 2014 Jun;43(3):655-65.

14. Morey VM, Song YD, Whang JS, Kang YG, Kim TK. Can serum albumin level and total lymphocyte count be surrogates for malnutrition to predict wound complications after total knee arthroplasty? J Arthroplasty. 2016 Jun;31(6):1317-21.

15. Hirasawa K, Tsukahara K, Motohashi R, Endo M, Sato H, Ueda Y, et al. Deep neck cellulitis: limitations of conservative treatment with antibiotics. Acta Otolaryngol. 2017 Jan;137(1):86-9.

16. Olejniczak I, Bojanowska-Pozniak K, Pietruszewska W. Deep neck infections: still important diagnostic and therapeutic problem. Otolaryngol Pol. 2016 Apr;70(2):25-30.

17.Wall DB, Klein SR, Black S, de Virgilio C.A simple model to help distinguish necrotizing fasciitis from nonnecrotizing soft tissue infection. J Am Coll Surg. 2000 Sep;191(3):227-31.

18. Celakovsky P, Kalfert D, Tucek L, Mejzlik J, Kotulek M, Vrbacky A, et al. Deep neck infections: risk factors for mediastinal extension. Eur Arch Otorhinolaryngol. 2014 Jun;271(6):1679-83.

19. Gausepohl JS, Wagner JG. Survival from cervical necrotizing fasciitis. West J Emerg Med. 2015 Jan;16(1):172-4.

20. Boscolo-Rizzo P, Stellin M, Muzzi E, Mantovani M, Fuson R, Lupato V, et al. Deep neck infections: a study of 365 cases highlighting recommendations for management and treatment. Eur Arch Otorhinolaryngol. 2012 Apr;269(4):1241-9.

21. Staffieri C, Fasanaro E, Favaretto N, La Torre FB, Sanguin S, Giacomelli L, et al. Multivariate approach to investigating prognostic factors in deep neck infections. Eur Arch Otorhinolaryngol. $2014 \mathrm{Jul}$; 271(7):2061-7.

22. Obregon-Guerrero G, Martinez-Ordaz JL, Moreno-Aguilera E, Ramirez-Martinez M, Pena-Garcia JF, Perez-Alvarez C. Deep neck abscess: factors related to reoperation and mortality. Cir Cir. 2013 Jul-Aug;81(4):299-306.

23. Sandner A, Moritz S, Unverzagt S, Plontke SK, Metz D. Cervical necrotizing fasciitis: the value of the laboratory risk indicator for necrotizing fasciitis score as an indicative parameter. J Oral Maxillofac Surg. 2015 Dec;73(12):2319-33.

24. Barber BR, Dziegielewski PT, Biron VL, Ma A, Seikaly H. Factors associated with severe deep neck space infections: targeting multiple fronts. J Otolaryngol Head Neck Surg. 2014 Aug;43(1):35.

25. Cardenas-Malta KR, Cortes-Flores AO, Fuentes-Orozco C, MartinezOropeza Ldel C, Lopez-Ramirez MK, Gonzalez-Ojeda A. Necrotizing mediastinitis in deep neck infections. Cir Cir. 2005 Jul-Aug;73(4): 263-7.

26. Cheng Z, Yu J, Xiao L, Lian Z, Wei Y, Wang J. Deep neck infection: clinical analyses of 95 cases. Zhonghua Er Bi Yan Hou Tou Jing Wai Ke Za Zhi. 2015 Sep;50(9):769-72.

27. Huang TT, Tseng FY, Yeh TH, Hsu CJ, Chen YS. Factors affecting the bacteriology of deep neck infection: a retrospective study of 128 patients. Acta Otolaryngol. 2006 Apr;126(4):396-401.

28. Yang W, Hu L, Wang Z, Nie G, Li X, Lin D, et al. Deep neck infection: a review of 130 cases in southern China. Medicine (Baltimore). 2015 Jul;94(27):e994.

29. Thomas AJ, Meyer TK. Retrospective evaluation of laboratory-based 
diagnostic tools for cervical necrotizing fasciitis. Laryngoscope. 2012 Dec;122(12):2683-7.

30.TsaiYH, Huang KC, Shen SH, HsuWH, Peng KT, Huang TJ. Microbiology and surgical indicators of necrotizing fasciitis in a tertiary hospital of southwest Taiwan. Int J Infect Dis. 2012 Mar;16(3):e159-65.

31. Allingstrup MJ, Esmailzadeh N, Wilkens Knudsen A, Espersen K, Hartvig Jensen T, Wiis J, et al. Provision of protein and energy in relation to measured requirements in intensive care patients. Clin Nutr. 2012 Aug;31(4):462-8.

32. Plank LD, Connolly AB, Hill GL. Sequential changes in the metabolic response in severely septic patients during the first 23 days after the onset of peritonitis. Ann Surg. 1998 Aug;228(2):146-58.

33. Geng Y, Qi Q, Sun M, Chen H, Wang P, Chen Z. Prognostic nutritional index predicts survival and correlates with systemic inflammatory response in advanced pancreatic cancer. Eur J Surg Oncol.
2015 Nov;41(11):1508-14

34. Casaer MP,Wilmer A, Hermans G, Wouters PJ, Mesotten D, Van den Berghe G. Role of disease and macronutrient dose in the randomized controlled EPaNIC trial: a post hoc analysis. Am J Respir Crit Care Med. 2013 Feb;187(3):247-55.

35. Jones JM. The methodology of nutritional screening and assessment tools. J Hum Nutr Diet. 2002 Feb;15(1):59-71.

36. Kondrup J, Allison SP, Elia M, Vellas B, Plauth M; Educational and Clinical Practice Committee, et al. ESPEN guidelines for nutrition screening 2002. Clin Nutr. 2003 Aug;22(4):415-21.

37. Kondrup J, Rasmussen HH, Hamberg O, Stanga Z; Ad Hoc ESPEN Working Group. Nutritional risk screening (NRS 2002): a new method based on an analysis of controlled clinical trials. Clin Nutr. 2003 Jun;22(3):321-36. 